\title{
Solution of Time Periodic Electroosmosis Flow with Slip Boundary
}

\author{
Qian Sun, ${ }^{1}$ Yonghong Wu, ${ }^{1}$ Lishan Liu, ${ }^{2}$ and B. Wiwatanapataphee ${ }^{3}$ \\ ${ }^{1}$ Department of Mathematics and Statistics, Curtin University, Perth, WA 6845, Australia \\ ${ }^{2}$ School of Mathematical Sciences, Qufu Normal University, Qufu, Shandong 273165, China \\ ${ }^{3}$ Department of Mathematics, Faculty of Science, Mahidol University, Bangkok 10400, Thailand
}

Correspondence should be addressed to Qian Sun; julysunqian@gmail.com and B.Wiwatanapataphee; benchawan.wiw@mahidol.ac.th

Received 27 March 2014; Revised 28 April 2014; Accepted 22 May 2014; Published 16 June 2014

Academic Editor: Xinguang Zhang

Copyright (C) 2014 Qian Sun et al. This is an open access article distributed under the Creative Commons Attribution License, which permits unrestricted use, distribution, and reproduction in any medium, provided the original work is properly cited.

Recent research confirms that slip of a fluid on the solid surface occurs at micrometer scale. Slip on solid surface may cause the change of interior material deformation which consequently leads to the change of velocity profile and stress field. This paper concerns the time periodic electroosmotic flow in a channel with slip boundary driven by an alternating electric field, which arises from the study of particle manipulation and separation such as flow pumping and mixing enhancement. Although exact solutions to various flow problems of electroosmotic flows under the no-slip condition have been obtained, exact solutions for problems under slip boundary conditions have seldom been addressed. In this paper, an exact solution is derived for the time periodic electroosmotic flow in two-dimensional straight channels under slip boundary conditions.

\section{Introduction}

One of the major focuses in scientific research during the past decades has been the study of material behaviour at microand nanoscale. The subject of micro- and nanofluidics concerns mass and momentum transfer in micro- and nanoscale systems or around micro- or nanosized objects, as well as the design and application of biological and engineering devices and systems involving fluid flow through microchannels [13]. The study of microflows has attracted great attention from the science and engineering communities in the effort of developing a better understanding of the mechanism of microflows and consequently developing better models and control. Traditionally, in the model for fluid flows, the no-slip boundary condition is used; that is, the fluid velocity relative to the solid is assumed to be zero on the fluid-solid interface. However, it has been established that the no-slip condition is a hypothesis instead of a condition deduced from any principle. Evidence of slip of a fluid on the solid surface was reported by many researchers $[4,5]$.

In recent years, time periodic electroosmosis has been studied intensively due to the recent development of microfluidic devices. Time periodic electroosmosis is known as $\mathrm{AC}$ electroosmosis, and the driving force is generated by an alternative electric field. Dose and Guiochon published numerical results for impulsive started electroosmotic flow [6]. Green et al. studied the electroosmosis on a planner microelectrode under both steady and unsteady fields [7]. Soderman and Jonsson investigated the electroosmotic flows for various geometries and derived various analytical results for the flow in two-dimensional microchannels and for the flow over a flat plate [8]. By using the green function method, Kang and coworkers [9] analysed the instantaneous velocity of the flow through a microchannel with time-dependent electric field. Jian et al. [10] studied the time periodic electroosmotic flow through circular microchannels. They discussed two different kinds of situations, which are the electroosmotic flow through the parallel plates and circular tubes.

Although analytical and numerical solutions to many flow problems of Newtonian fluids have been established under the no-slip assumption [11-13], very few exact solutions for time-dependent problems have been obtained for the slip 


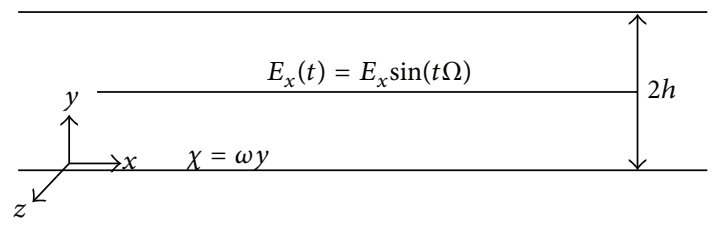

FIGURE 1: The flow channel and coordinate system used.

case $[14,15]$. Hence, we investigate, in this paper, the time periodic electroosmotic flows in a microchannel under the Navier slip assumption. The rest of the paper is organized as follows. In Section 2, the mathematical equations for the channel flow are presented, which include the partial differential equations and the boundary conditions based on the slip assumption. In Section 3, the general solution for the velocity of the fluid in the channel is derived with some arbitrary constants. Then the slip boundary condition and symmetric condition are used to determine the arbitrary constants. Numerical investigations are then given in Section 4, followed by a concluding remark in Section 5 .

\section{Problem Description and Mathematical Formation}

The problem considered is the electrically driven flow of fluid through channels with height $2 h$. The geometry of the channels and the coordinates $(x, y, z)$ used are shown in Figure 1.

Based on the principle of continuous mechanics, the governing equations for the flow of an incompressible ionized fluid are the standard Navier-Stokes equations:

$$
\rho_{f}\left(\frac{\partial \vec{V}}{\partial t}+(\vec{V} \cdot \nabla) \vec{V}\right)=-\nabla P+\mu \nabla^{2} \vec{V}+\rho_{e} \vec{E}
$$

where $P$ is the pressure, $\rho_{f}$ and $\rho_{e}$ are, respectively, the fluid density and the electric charge density, and $\vec{V}=(u, v, w)$ is the velocity vector with $\mu$ being the fluid viscosity. The last term on the right side denotes the electroosmotic body forces and $\vec{E}$ denotes the externally applied electric field.

We assume fully developed symmetric channel flow, and thus the swirling flow and consequently the velocity components in $y$ and $z$ directions can be neglected. This allows us to use the superposition principle because the resulted unsteady Navier-Stokes equations for this case are linear in velocity. This means that we can solve exactly the pressure and electroosmotically driven flows separately and then combine the two solutions to get the solution of the problem with the combined effects. The form of the electroosmotic body force, in general, depends on the form of the electric field applied on the system. In this paper, we will focus on sinusoidally driven flows.

Taking into account the above assumptions, (1) reduces to

$$
\rho_{f} \frac{\partial u}{\partial t}=\mu \frac{\partial^{2} u}{\partial y^{2}}+\rho_{e} E_{x} \sin (\Omega t)
$$

where $E_{x}$ and $\Omega$ are, respectively, the magnitude and the frequency of the external electric field $\vec{E}$. With (2), we can then determine the velocity of the flow driven by the time periodic electric field.

Based on the theory of electric field [16], the $\rho_{e}$ in (2) can be determined by

$$
\rho_{e}=-2 n_{0} e z \sinh \left(\frac{e z \psi}{k_{B} T}\right),
$$

where $n_{0}$ denotes the average number of positive or negative ions in the buffer, $z$ is the valance, $e$ is the electron charge, $T$ is the absolute temperature, $\psi$ is the electric potential, and $k_{B}$ is the Boltzmann constant. Let $\psi^{*}=\psi / \xi$ be the normalized electroosmotic potential in which $\xi$ is the zeta potential and let $\alpha=\left(e z \xi / k_{B}\right) T$ be a parameter; then we have

$$
\rho_{e}=-2 n_{0} e z \sinh \left(\alpha \psi^{*}\right) \text {. }
$$

Based on the work in [16], $\psi$ is governed by the PoissonBoltzmann equation:

$$
\nabla^{2} \psi=-\frac{\rho_{e}}{\epsilon} .
$$

in which $\epsilon$ is the permittivity, and we have assumed that the effect of time fluctuation of the external electric field on the electric potential is negligible.

It has been established in the literature that the effective thickness of the electric double layer (EDL) depends on the ionic energy parameter $\alpha$. For example, for $\alpha=5$, the effective electric double layer thickness is about $4.217 \lambda$, where $\lambda$ is related to other system parameters by

$$
\omega=\frac{1}{\lambda}=\sqrt{\frac{8 \pi n_{0} e^{2} z^{2}}{D k_{B} T}} .
$$

In most microfluidic applications, the value of $\lambda$ is about $10-100 \mathrm{~nm}$, which is generally much smaller than the height of a typical microchannel. Hence, the electroosmotic potential generally reaches zero in the channel center, and its distribution along the channel height can be expressed by $[16,17]$

$$
\psi^{*}=\frac{4}{\alpha} \tanh ^{-1}\left[\tanh \left(\frac{\alpha}{4}\right) \exp (-\chi)\right]
$$

where $\chi=y / \lambda$ denotes the distance from the channel wall. From the definition of the Debye-Huckel parameter $\omega$, we can write the electroosmotic body force as

$$
-2 n_{0} e z E_{x} \sin \Omega t=\frac{\omega^{2} \mu u_{\mathrm{HS}}}{\alpha} \sin \Omega t,
$$

where $u_{\mathrm{HS}}=-\epsilon \zeta E_{x} / \mu$ is the Helmholtz-Smoluchowski velocity. Then, (2) can be written as

$$
\rho_{f} \frac{\partial u}{\partial t}=\mu \frac{\partial^{2} u}{\partial y^{2}}+\frac{\omega^{2} \mu u_{\mathrm{HS}}}{\alpha} \sinh \left(\alpha \psi^{*}\right) \sin \Omega t .
$$

To completely determine the fluid flow in the channel, the differential equation (2) must be supplemented by a 
TABLE 1: $A$ and $B$ values for different sets of model parameters.

\begin{tabular}{llrr}
\hline$\kappa$ & $\alpha$ & $A$ & $B$ \\
\hline \multirow{2}{*}{0.1} & 1 & $-1.5583-1.6348 i$ & $-4.6373+2.0952 i$ \\
& 3 & $-18.0859-2.6534 i$ & $-23.1309+3.2856 i$ \\
& 5 & $-23.6809-3.3261 i$ & $-30.0499+4.0098 i$ \\
0.01 & 1 & $23.9849-37.1567 i$ & $-34.8651+38.3333 i$ \\
& 3 & $38.9742-59.1730 i$ & $-57.6358+60.8270 i$ \\
& 5 & $10.9090-537.4980 i$ & $-104.6609+610.5019 i$ \\
0.001 & 1 & $372.8097-389.5891 i$ & $-385.0603+389.7408 i$ \\
& 3 & $593.6919-619.3798 i$ & $-611.3080+619.6202 i$ \\
& 5 & $732.7149-764.5012 i$ & $-754.4851+764.7988 i$ \\
\hline
\end{tabular}

set of boundary and initial conditions. To describe the slip characteristics of a fluid on the solid surface, the Navier slip condition is used; namely, the velocity of fluid, relative to the solid surface, is assumed to be proportional to the sheer stress on the fluid-solid surface; that is,

$$
u(0, t)+l \frac{\partial u}{\partial y}(0, t)=0
$$

where $l$ is the so-called slip length. We should address here that, for $l=0$, condition (10) reduces to the no-slip boundary condition.

In addition, due to the symmetry of velocity about the symmetric plane $y=h$, we have

$$
\frac{\partial u}{\partial y}(h, t)=0
$$

Now the partial differential equation (9) together with conditions (10) and (11) constitutes a complete boundary value problem for the fluid flow in the channel.

\section{Solution of the Boundary Value Problems}

By introducing the characteristic time $1 / \Omega$ and the characteristic length $\lambda$, (9) can be expressed in normalized form

$$
\rho_{f} u_{\mathrm{HS}} \Omega \frac{\partial U}{\partial \theta}=\mu u_{\mathrm{HS}} \omega^{2}\left[\frac{\partial^{2} U}{\partial^{2} \chi}+\frac{\sin (\theta)}{\alpha} \sinh \left(\alpha \psi^{*}\right)\right],
$$

in which $\theta=\Omega t$ denotes the nondimensional time, $\chi=$ $y / \lambda$ denotes the nondimensional distance, and $U=u / u_{\mathrm{HS}}$ denotes the nondimensional velocity. From (12), we have

$$
\frac{\partial U}{\partial \theta}=\frac{1}{\kappa^{2}}\left[\frac{\partial^{2} U}{\partial^{2} \chi}+\frac{\sin (\theta)}{\alpha} \sinh \left(\alpha \psi^{*}\right)\right],
$$

where $\kappa=\left(\Omega \lambda^{2} / \mu\right)^{1 / 2}$ is a normalized parameter expressed as a function of the Debye length $(\lambda)$, the electric field excitation frequency $(\Omega)$, and the kinematic viscosity $(\mu)$. The parameter $\kappa$ represents the ratio of the Debye length to a diffusion length scale $\left(I_{D}\right)$ given by

$$
I_{D}=\frac{\lambda}{\kappa} .
$$

Let $\widetilde{U}$ be the solution of the following equation:

$$
\frac{\partial \widetilde{U}}{\partial \theta}=\frac{1}{\kappa^{2}}\left[\frac{\partial^{2} \widetilde{U}}{\partial^{2} \chi}+\frac{e^{i \theta}}{\alpha} \sinh \left(\alpha \psi^{*}\right)\right] .
$$

Then, from the linearity and the superposition principle, the solution of (13) will be $U=\operatorname{Im}(\widetilde{U})$. It is noted that (15) admits solution of the form $\widetilde{U}=e^{i \theta} F(\chi)$ where we have written $\sin (\theta)$ as the imaginary part of $\exp (i \theta)$, and thus we proceed to solve (15) first.

The derivative of $U(\chi, \theta)$ with respect to time can then be written as

$$
\frac{\partial \widetilde{U}}{\partial \theta}=i \exp (i \theta) F(\chi)
$$

and the second space derivatives can be written as

$$
\frac{\partial^{2} \widetilde{U}}{\partial^{2} \chi}=\exp (i \theta) \frac{d^{2} F}{d \chi^{2}}
$$

Therefore (15) becomes

$$
i \exp (i \theta) F(\chi)=\frac{\exp (i \theta)}{\kappa^{2}}\left[\frac{d^{2} F}{d \chi^{2}}+\frac{\sinh \left(\alpha \psi^{*}\right)}{\alpha}\right] .
$$

For the above to be true for all $\theta$ values, we require

$$
\frac{d^{2} F(\chi)}{d \chi^{2}}-i \kappa^{2} F(\chi)=-\frac{\sinh \left(\alpha \psi^{*}\right)}{\alpha} .
$$

The general solution of (19) includes a complimentary solution and a particular solution. The complimentary solution can be derived by solving the associated homogenous equation, while the particular solution can be obtained by using the variation of parameter methods. Through some calculation, we have

$$
\begin{aligned}
F(\chi)= & A \exp (\kappa \chi \sqrt{i})+B \exp (-\kappa \chi \sqrt{i}) \\
& +\frac{1}{2 \kappa \alpha \sqrt{i}}[\exp (-\kappa \chi \sqrt{i})
\end{aligned}
$$



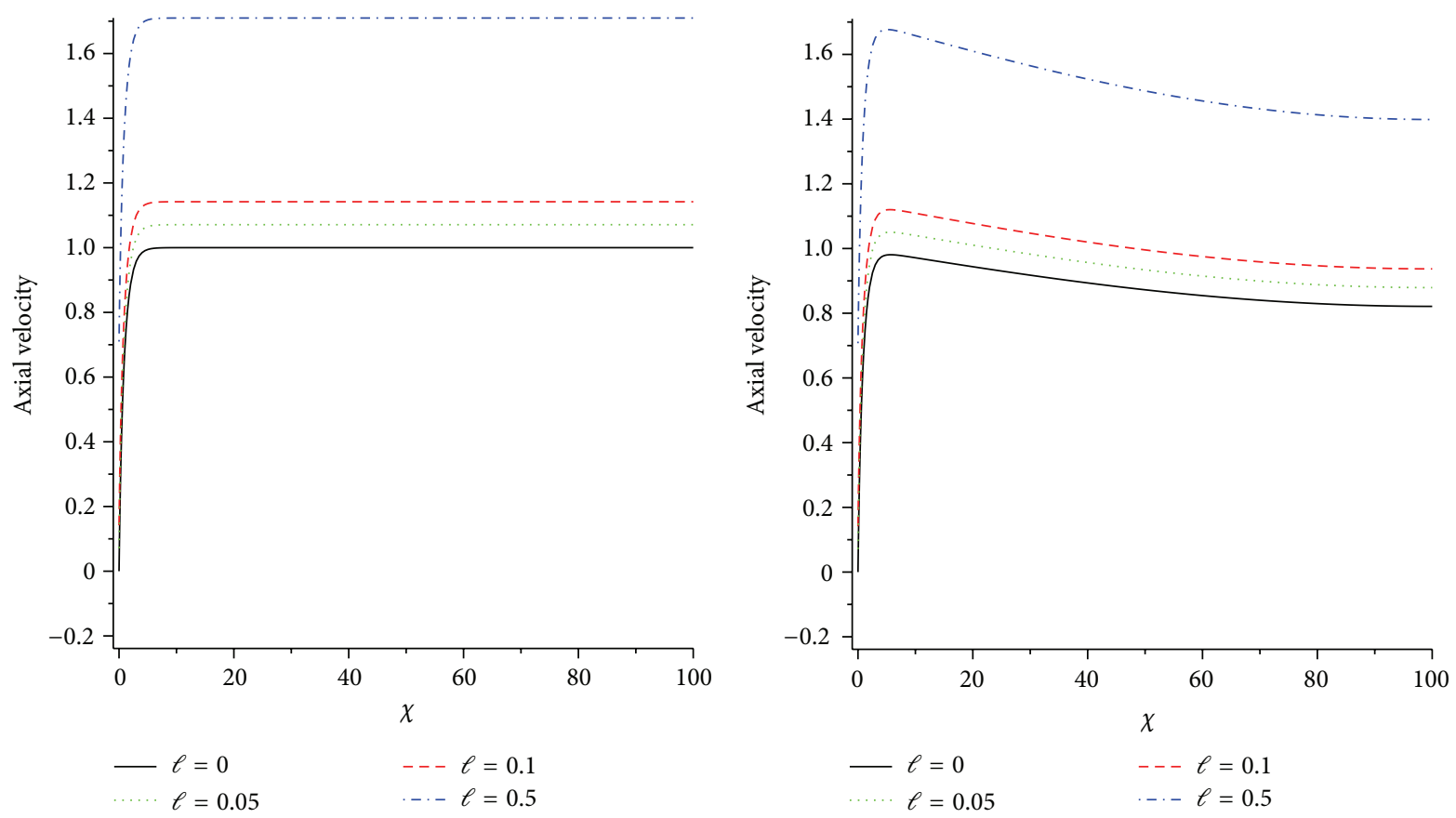

(a) $\kappa=0.001$
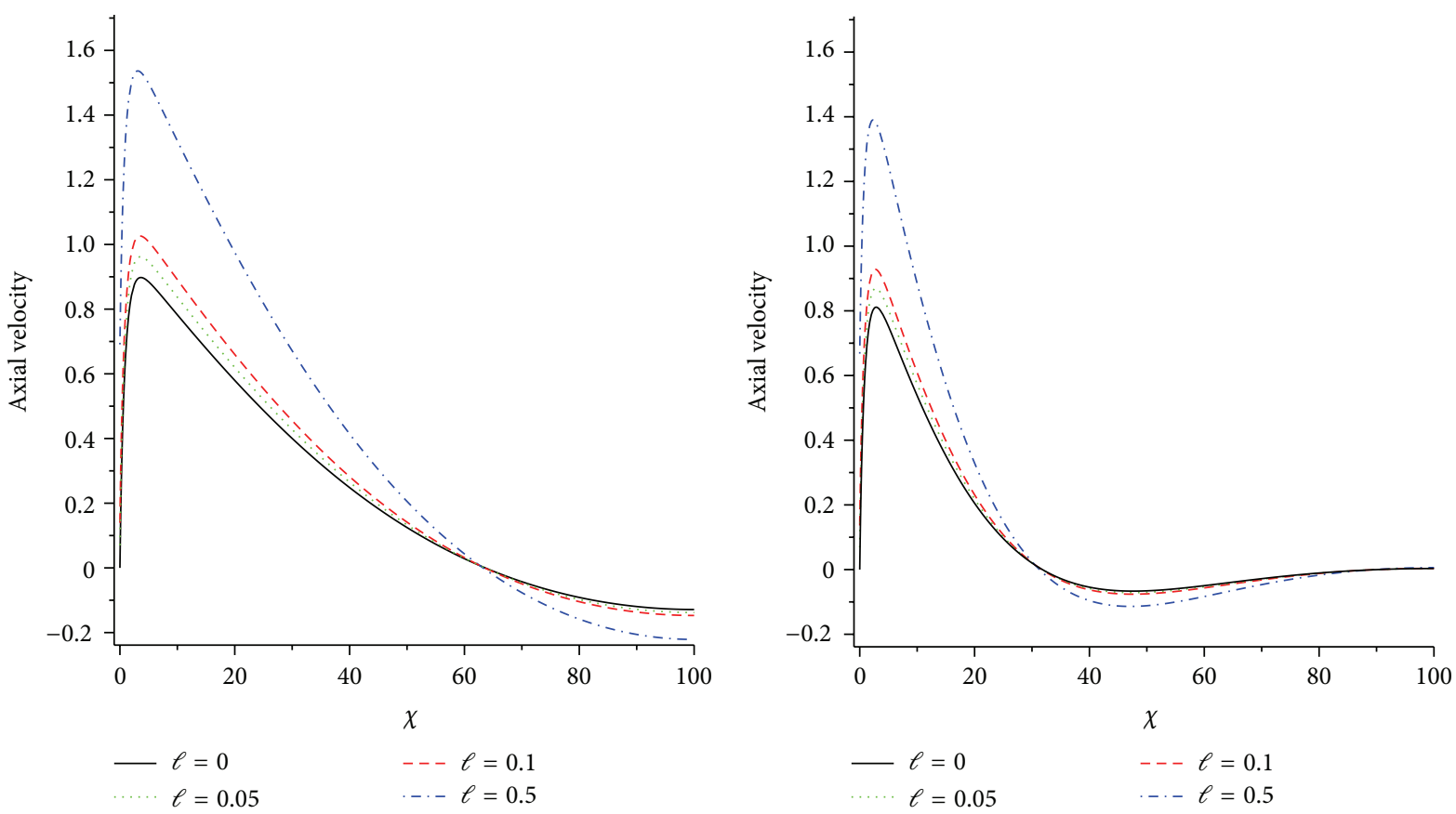

(c) $\kappa=0.03$

(d) $\kappa=0.07$

FIGURE 2: Effect of slip length on velocity profile obtained from the model with $\theta=\pi / 2, \alpha=1$, and $h=100 \lambda$ for various $\kappa$ values: (a) $\kappa=0.001$; (b) $\kappa=0.01$; (c) $\kappa=0.03$; (d) $\kappa=0.07$.

$$
\begin{array}{r}
\left.\times \int_{0}^{\chi} \exp (\kappa \chi \sqrt{i}) \sinh \left(\alpha \psi^{*}\right) d \chi\right] \\
-\frac{1}{2 \kappa \alpha \sqrt{i}} \exp (\kappa \chi \sqrt{i}) \\
\times \int_{0}^{\chi} \exp (-\kappa \chi \sqrt{i}) \sinh \left(\alpha \psi^{*}\right) d \chi
\end{array}
$$

and consequently we have

$$
\begin{aligned}
& \widetilde{U}(\chi, \theta)= A \exp (i \theta) \exp (\kappa \chi \sqrt{i})+B \exp (i \theta) \exp (-\kappa \chi \sqrt{i}) \\
&+\frac{\exp (i \theta)}{2 \kappa \alpha \sqrt{i}}[\exp (-\kappa \chi \sqrt{i}) \\
&\left.\times \int_{0}^{\chi} \exp (\kappa \chi \sqrt{i}) \sinh \left(\alpha \psi^{*}\right) d \chi\right]
\end{aligned}
$$




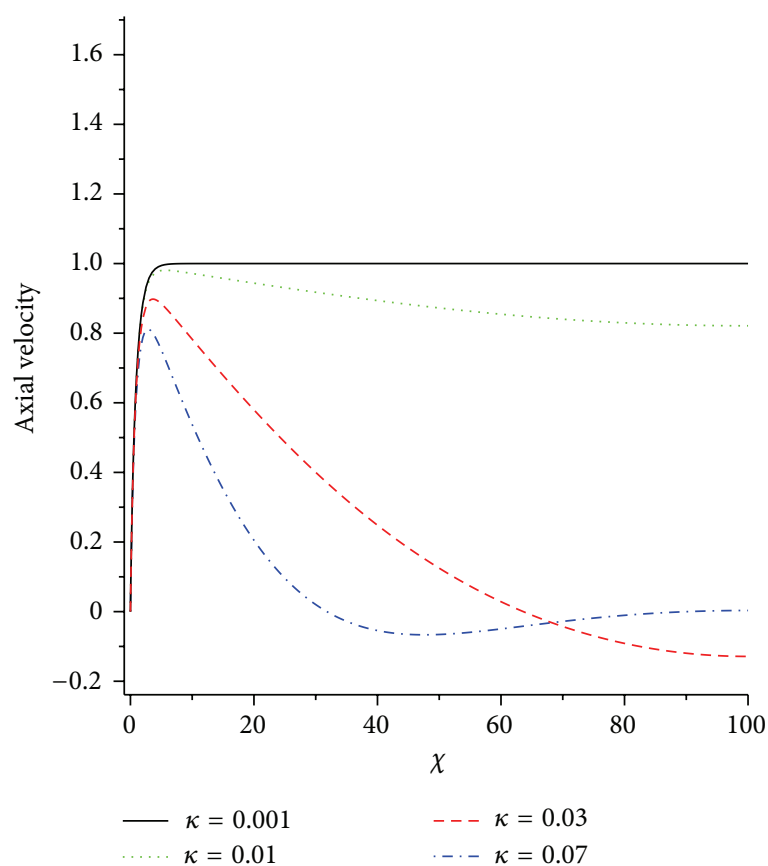

(a) $\ell=0$

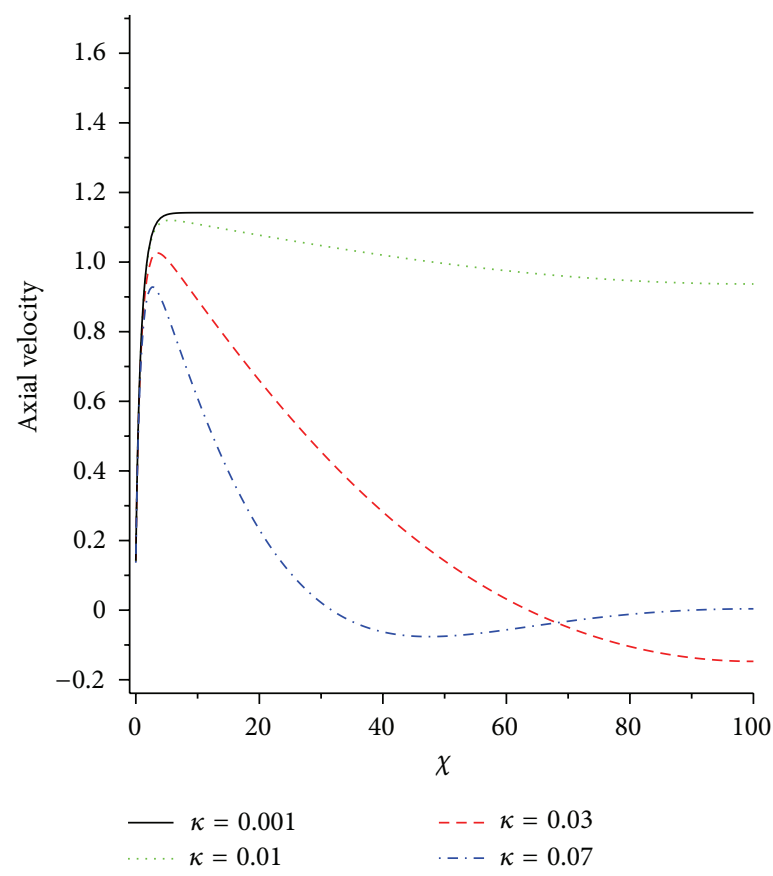

(c) $\ell=0.1$

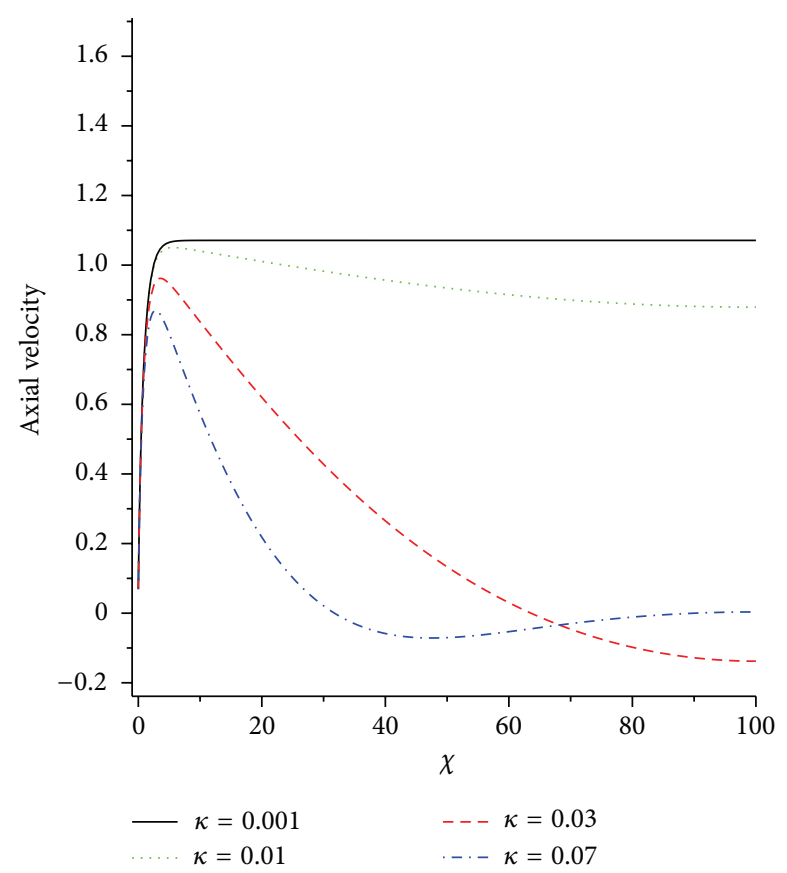

(b) $\ell=0.05$

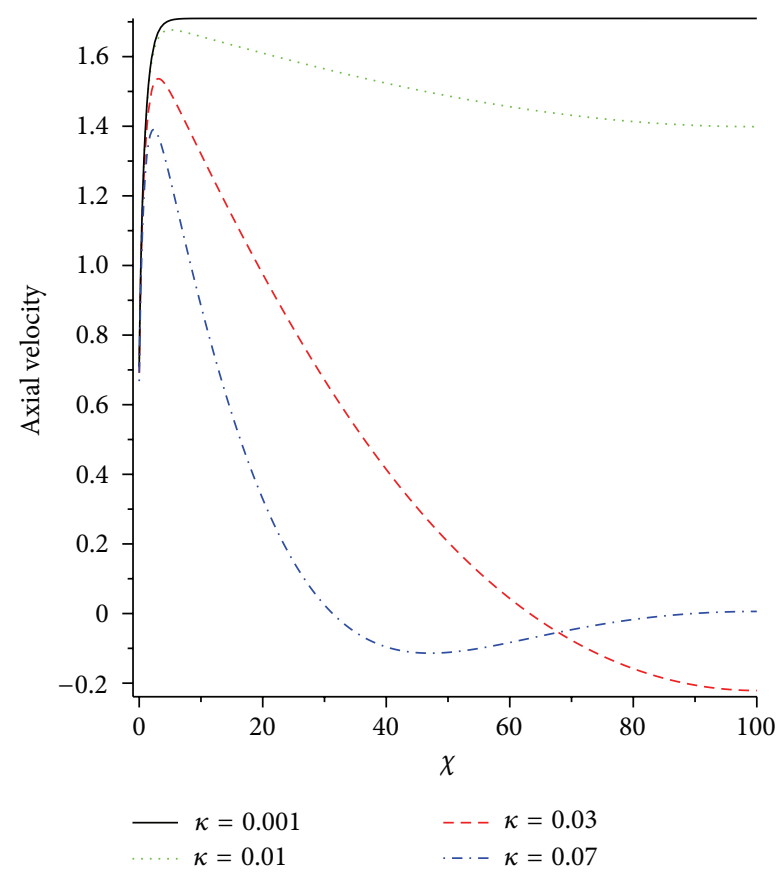

(d) $\ell=0.5$

FIGURE 3: Effect of $\kappa$ on velocity profile obtained from the model with $\theta=\pi / 2, \alpha=1$, and $h=100 \lambda$ for various slip lengths: (a) $\ell=0$; (b) $\ell=0.05 ;$ (c) $\ell=0.1 ;$ (d) $\ell=0.5$.

$$
\begin{aligned}
-\frac{\exp (i \theta)}{2 \kappa \alpha \sqrt{i}}\left[\exp (\kappa \chi \sqrt{i}) \int_{0}^{\chi}\right. & \exp (-\kappa \chi \sqrt{i}) \\
& \left.\times \sinh \left(\alpha \psi^{*}\right) d \chi\right] .
\end{aligned}
$$

Now we proceed to determine the arbitrary constants in (21). In terms of the nondimensional time $\theta$, the nondimen- sional distance $\chi$, and the nondimensional velocity $U$, the boundary conditions (10) and (11) become

$$
\begin{aligned}
& U(0, \theta)+\frac{l}{\lambda} \frac{\partial U}{\partial \chi}(0, \theta)=0 \\
& \frac{\partial U}{\partial \chi}\left(\frac{h}{\lambda}, \theta\right)=0
\end{aligned}
$$




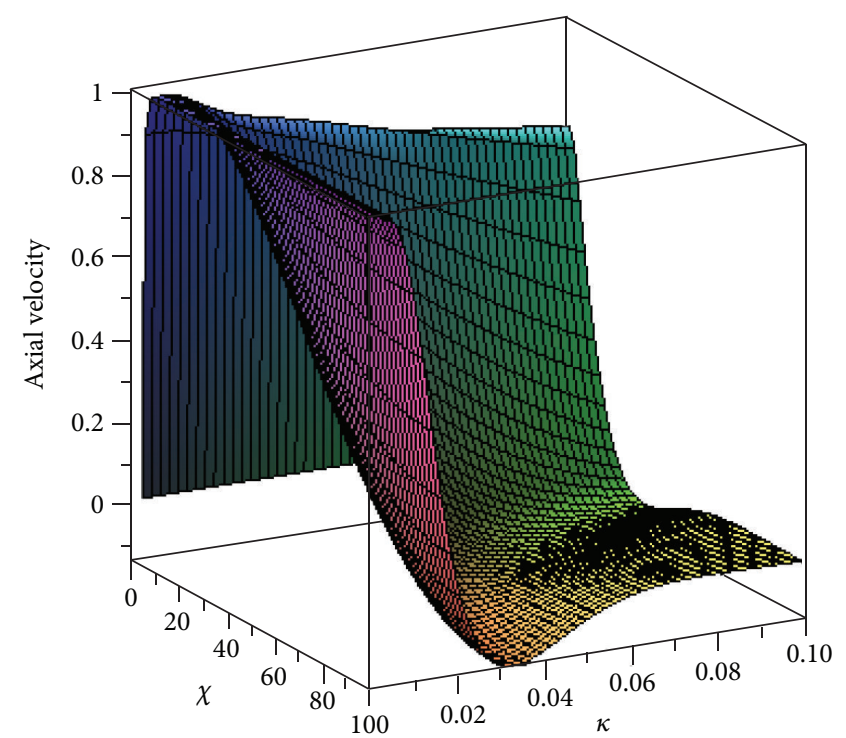

(a) $U(\chi, \kappa)$ when $\ell=0.05$

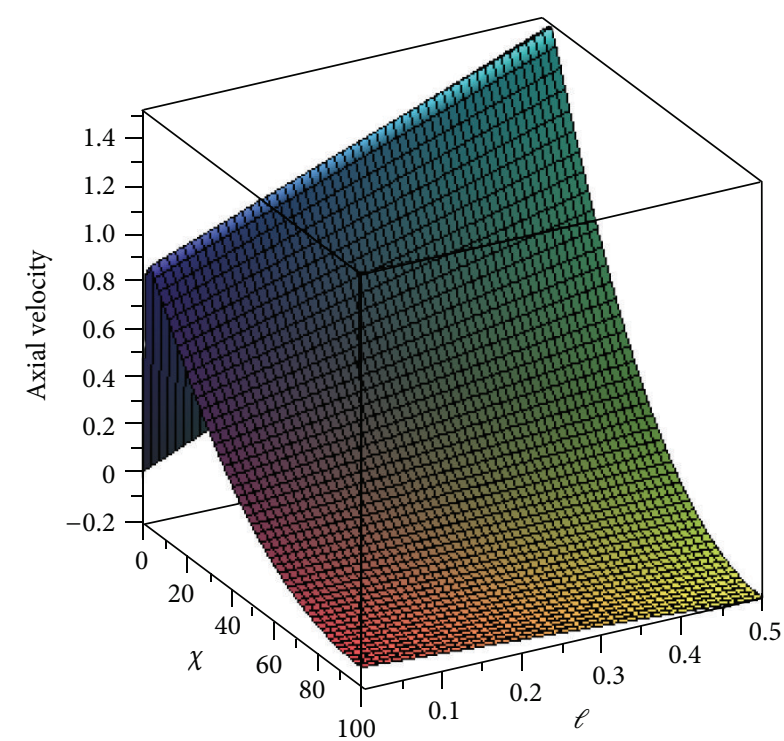

(b) $U(\chi, l)$ when $\kappa=0.03$

Figure 4: 3D plot of $U(\chi, \kappa)$ and $U(\chi, l)$ obtained from the model with $\theta=\pi / 2, \alpha=1$, and $h=100 \lambda$.

As $U(\chi, \theta)=\operatorname{Im}(\widetilde{U}(\chi, \theta)),(22)$ will be satisfied if $\widetilde{U}$ satisfies the following boundary conditions:

$$
\begin{aligned}
& \widetilde{U}(0, \theta)+\frac{l}{\lambda} \frac{\partial \widetilde{U}}{\partial \chi}(0, \theta)=0, \\
& \frac{\partial \widetilde{U}}{\partial \chi}\left(\frac{h}{\lambda}, \theta\right)=0 .
\end{aligned}
$$

Noticing that $\widetilde{U}=e^{i \theta} F(\chi)$, the above boundary conditions become

$$
\begin{aligned}
& F(0)+\frac{l}{\lambda} F^{\prime}(0)=0, \\
& F^{\prime}\left(\frac{h}{\lambda}\right)=0 .
\end{aligned}
$$

To apply the boundary condition (24), we first need to calculate the spatial derivative of $F(\chi)$. From (7) and (20), we have

$$
\begin{gathered}
F^{\prime}(\chi)=A(\kappa \sqrt{i}) \exp (\kappa \chi \sqrt{i})+B(-\kappa \sqrt{i}) \exp (-\kappa \chi \sqrt{i}) \\
-\frac{1}{2 \alpha}\left[\exp (-\kappa \chi \sqrt{i}) \int_{0}^{\chi} \exp (\kappa \chi \sqrt{i})\right. \\
\left.\times \sinh \left(\alpha \psi^{*}\right) d \chi\right] \\
-\frac{1}{2 \alpha}\left[\exp (\kappa \chi \sqrt{i}) \int_{0}^{\chi} \exp (-\kappa \chi \sqrt{i})\right. \\
\left.\times \sinh \left(\alpha \psi^{*}\right) d \chi\right] .
\end{gathered}
$$

At the centre of the channel, $\chi=h / \lambda$ and $d F / d \chi=0$ and so from (24) and (25) we have

$$
\begin{aligned}
& F^{\prime}\left(\frac{h}{\lambda}\right)= A(\kappa \sqrt{i}) \exp \left(\kappa \frac{h}{\lambda} \sqrt{i}\right)+B(-\kappa \sqrt{i}) \exp \left(-\kappa \frac{h}{\lambda} \sqrt{i}\right) \\
&- \frac{1}{2 \alpha} \exp \left(-\kappa \frac{h}{\lambda} \sqrt{i}\right) \int_{0}^{h / \lambda} \exp (\kappa \chi \sqrt{i}) \\
& \times \sinh \left(\alpha \psi^{*}\right) d \chi \\
&-\frac{1}{2 \alpha} \exp \left(\kappa \frac{h}{\lambda} \sqrt{i}\right) \int_{0}^{h / \lambda} \exp (-\kappa \chi \sqrt{i}) \\
& \times \sinh \left(\alpha \psi^{*}\right) d \chi=0 .
\end{aligned}
$$

From (20), (25), and the first equation of (24), we get

$$
\left(1+\frac{l}{\lambda} \kappa \sqrt{i}\right) A+\left(1-\frac{l}{\lambda} \kappa \sqrt{i}\right) B=0
$$

and hence

$$
B=-\left(1-\frac{l}{\lambda} \kappa \sqrt{i}\right)^{-1}\left(1+\frac{l}{\lambda} \kappa \sqrt{i}\right) A \text {. }
$$

Substituting (28) into (26) yields

$$
\begin{aligned}
A=\{ & \exp \left(\kappa \frac{h}{\lambda} \sqrt{i}\right)+\left(1-\frac{l}{\lambda} \kappa \sqrt{i}\right)^{-1} \\
& \left.\times\left(1+\frac{l}{\lambda} \kappa \sqrt{i}\right) \exp \left(-\kappa \frac{h}{\lambda} \sqrt{i}\right)\right\}^{-1}
\end{aligned}
$$



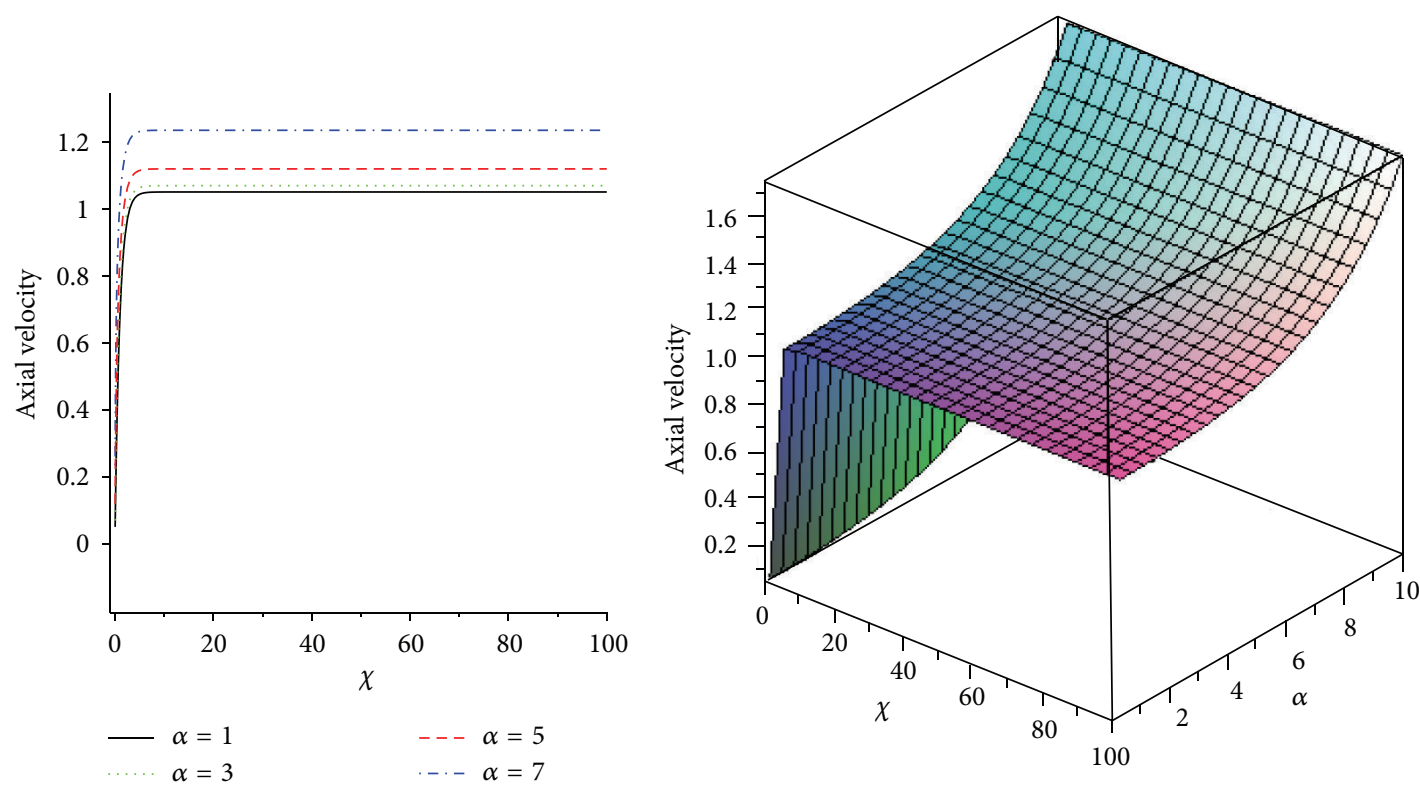

(a) $\kappa=0.001$
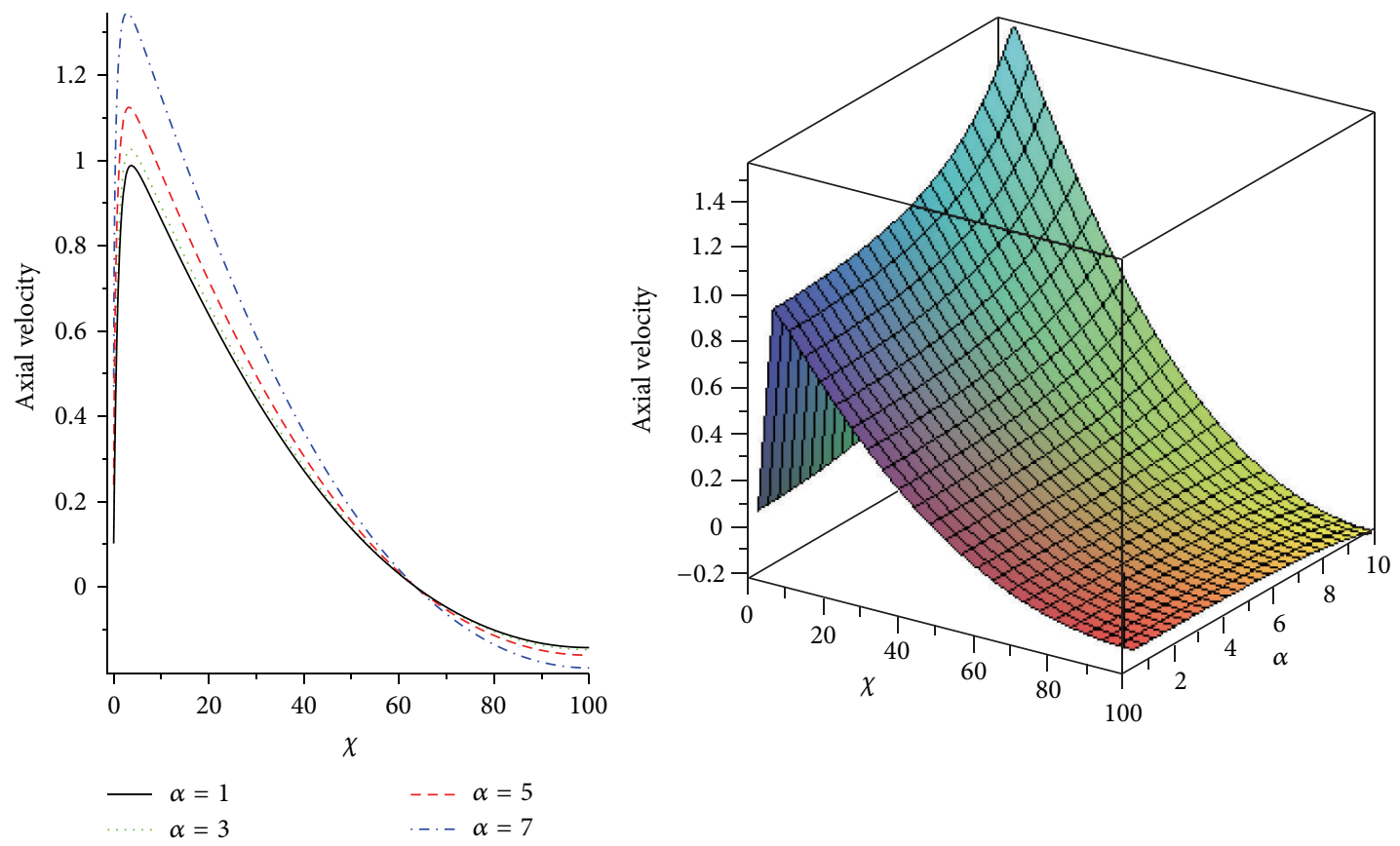

(b) $\kappa=0.03$

FiguRE 5: Effect of the energy parameter $\alpha$ on the velocity profile obtained from the model with $\theta=\pi / 2, h=100 \lambda$, and $l=0.05$ for two different $\kappa$ values: (a) $\kappa=0.001$ and (b) $\kappa=0.03$.

$$
\begin{aligned}
\times\left\{\frac{1}{2 \alpha \kappa \sqrt{i}} \exp \left(-\kappa \frac{h}{\lambda} \sqrt{i}\right) \int_{0}^{h / \lambda}\right. & \exp (\kappa \chi \sqrt{i}) \\
& \times \sinh \left(\alpha \psi^{*}\right) d \chi \\
+\frac{1}{2 \alpha \kappa \sqrt{i}} \exp \left(\kappa \frac{h}{\lambda} \sqrt{i}\right) \int_{0}^{h / \lambda} & \exp (-\kappa \chi \sqrt{i}) \\
\times & \left.\sinh \left(\alpha \psi^{*}\right) d \chi\right\} .
\end{aligned}
$$

\section{Numerical Investigation}

In this section, we investigate the characteristic of the electric driven flow. In particular, we will investigate the influence of various model parameters on the flow behaviour, including the $\kappa$ value, the energy parameter $\alpha$, the channel height $h$, and the slip parameter $l$. Table 1 shows the solutions of $A$ and $B$ for various sets of the model parameters.

In the first example of investigation, we set the channel height $2 h$ to be $200 \lambda$ where the $\lambda$ typically has value of 

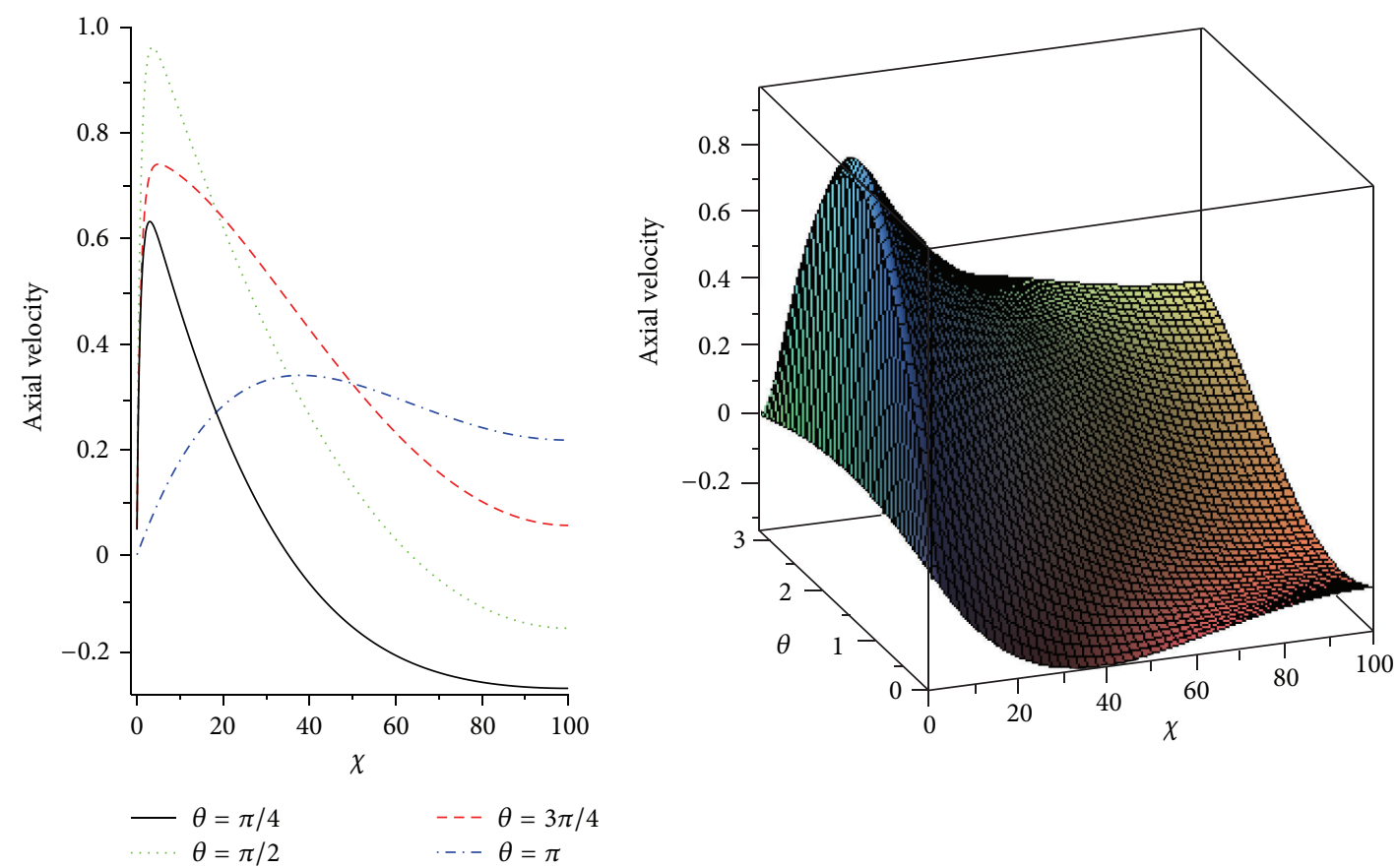

FIGURE 6: 2D and 3D plots of velocity profile at various instants of time obtained from the model with $\alpha=1, \kappa=0.03, \ell=0.05$, and $h=100 \lambda$.

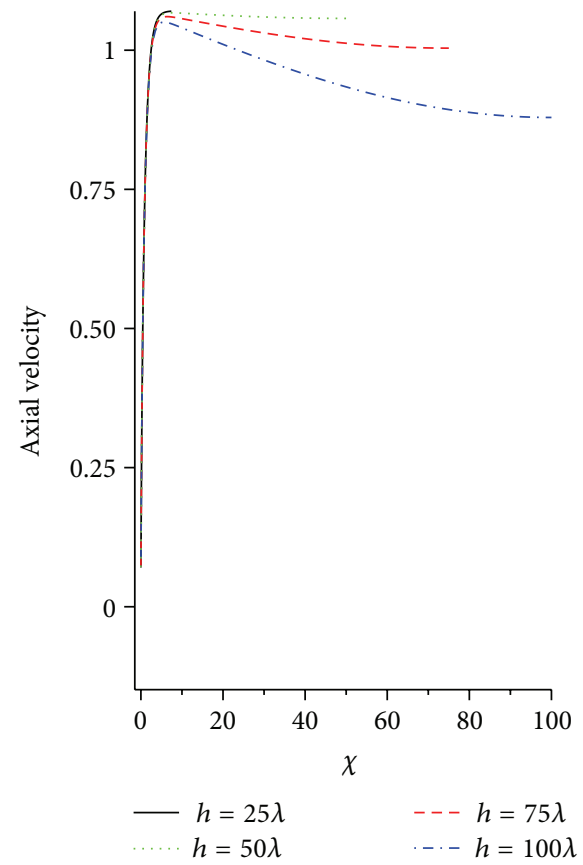

(a) $\kappa=0.001$

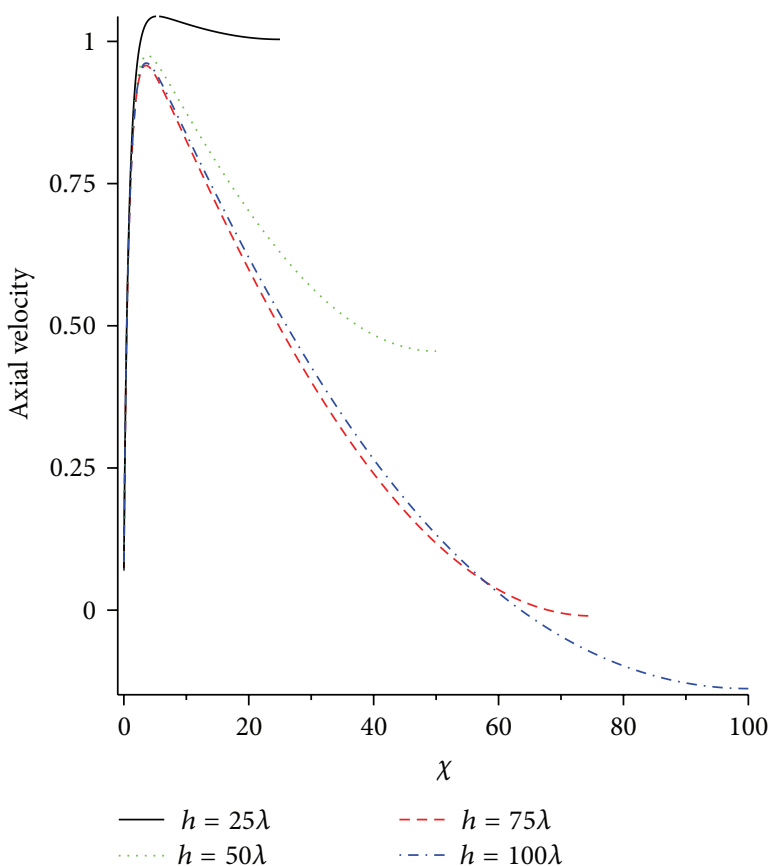

(b) $\kappa=0.03$

FIGURE 7: Effect of the channel half-height $h$ on the velocity profile obtained from the model with $\theta=\pi / 2, \alpha=1$, and $\ell=0.05$ for two different $\mathcal{K}$ values: (a) $\kappa=0.001$ and (b) $\kappa=0.03$.

hundreds of nanometres. Other parameters are chosen as $\alpha=1$ and $\lambda=1$. Figures 2 and 3 show the distribution of velocity along the channel height obtained from the model with various $l$ and $\kappa$ values at $\theta=\pi / 2$, as functions of $\chi$ for different values of the parameters $\kappa$ and $l$. Figure 4 presents the velocity distribution $U(\chi, \kappa)$ and $U(\chi, l)$, at $\theta=\pi / 2$ for $\alpha=1$ and $h=100 \lambda$. The results show that as $\kappa$ increases, the thickness of the layer with significant electrically driven flow decreases and essentially the velocity at the centre of the channel is zero for $\kappa \geq 0.07$ as shown in Figures 3(d) and 4(a). 


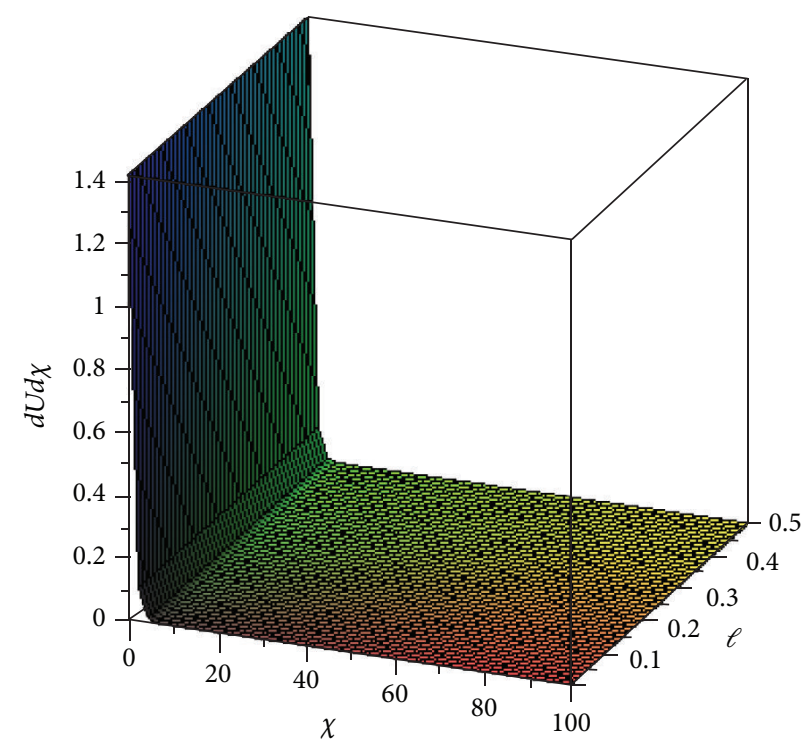

(a) $(D U / D \chi)(\chi, l)$ for $\kappa=0.001$

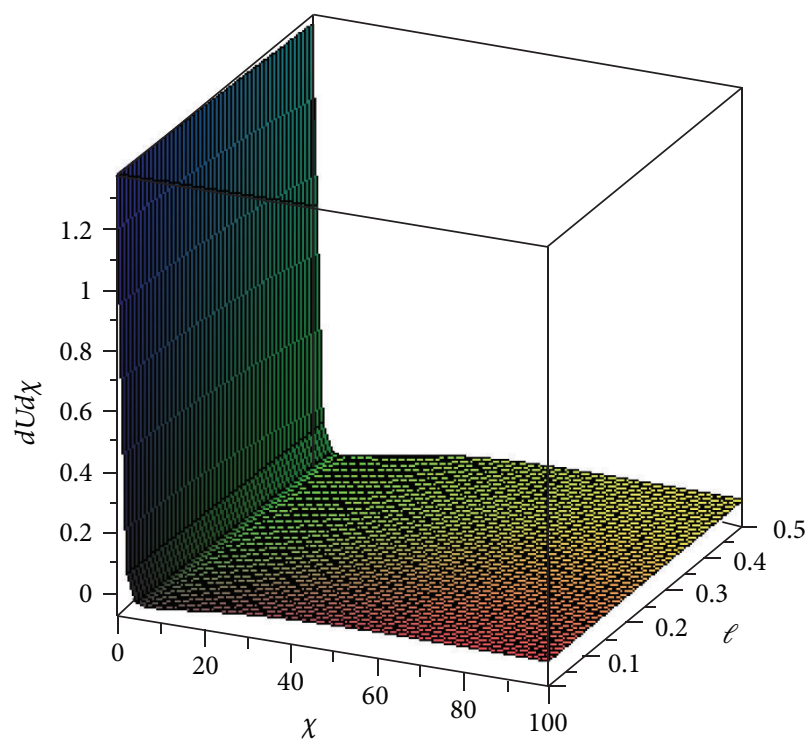

(b) $(D U / D \chi)(\chi, l)$ for $\kappa=0.03$

Figure 8: 3D plot of $(D U / D \chi)(\chi, l)$ for two $\kappa$ values: (a) $\kappa=0.001$ and (b) $\kappa=0.03$.

It is also found that the slip parameter has significant influence on the flow behaviour. At $\kappa=0.001$, as $l$ increases from 0 to 0.5 , the flow velocity increases from 1 to about 1.685 .

To investigate the effect of the energy parameter $\alpha$ on the flow behaviour, we choose four values of $\alpha$ including 1,3 , 5 , and 7. Figure 5 shows the influence of $\alpha$ on the velocity distribution for $\theta=\pi / 2, h=100 \lambda, l=0.05$ and two different $\kappa$ values of 0.001 and 0.03 . For a channel with fixed dimension, filled with a given buffer solution, the value of $\kappa$ depends directly on the frequency of the external electric field. Hence, in practice, it is possible to obtain the desired velocity field by controlling the frequency by electric switch [7]. We found that as $\alpha$ increases, the flow velocity increases. At $\kappa=0.001$, as $\alpha$ increases from 1 to 7 , the flow velocity at the centre increases from 1 to 1.4 as shown in Figure 5. At $\kappa=0.03$, as $\alpha$ increases from 1 to 7 , the flow at the centre approaches zero.

At the typical values of $\alpha=1, \kappa=0.03$, and $l=0.05$, the distributions of velocity along the height of the channel at various instants of time in an oscillation cycle of electric field are shown in Figure 6. The magnitude of the velocity varies with time, which is because of the fluctuation of the external electric field in time.

Figure 7 shows the influence of channel height on the magnitude of velocity. It can be noted that the magnitude of velocity is influenced by the channel height $h$, while the pattern of velocity profile along the channel is the same. Figure 8 shows the influence of $\kappa$ on the first derivative of the velocity.

\section{Concluding Results}

In this paper, we study the time periodic electroosmotic flow in a two-dimensional microchannel with slip boundary. Some analytical and numerical solutions for the slip velocity field under various different conditions have been obtained.
Our results show that the flow behaviour depends on the parameters $\kappa$ and $\alpha$ as well as the slip parameter $l$ and channel height. The parameter $\kappa$ represents the ratio of the electric double layer thickness to a characteristic diffusion length scale $I_{D}$, while $\alpha$ is an energy parameter.

We found that, depending on the value of the parameter $\kappa$, the flow pattern may change from oscillatory plug flow to uniform flat flow. Generally, as $\kappa$ increases, the velocity at the centre drops to zero dramatically. We also found that the slip parameter has significant influence on the flow behaviour. As $l$ increases, the flow rate increases.

\section{Conflict of Interests}

The authors declare that there is no conflict of interests regarding the publication of this paper.

\section{Acknowledgments}

Qian Sun, Yonghong Wu, and Lishan Liu were supported financially by the National Natural Science Foundation of China (11371221), the Program for Scientific Research Innovation Team in Colleges and Universities of Shandong Province, and the Specialized Research Foundation for the Doctoral Program of Higher Education of China (20123705110001).

\section{References}

[1] B. Bourlon, J. Wong, C. Mikó, L. Forró, and M. Bockrath, "A nanoscale probe for fluidic and ionic transport," Nature Nanotechnology, vol. 2, no. 2, pp. 104-107, 2007.

[2] H. Herwig and O. Hausner, "Critical view on new results in micro-fluid mechanics : an example," International Journal of Heat and Mass Transfer, vol. 46, no. 5, pp. 935-937, 2003. 
[3] H. Huang, T. S. Lee, and C. Shu, "Lattice Boltzmann method simulation gas slip flow in long microtubes," International Journal of Numerical Methods for Heat and Fluid Flow, vol. 17, no. 6, pp. 587-607, 2007.

[4] G. Chauveteau, "Rodlike polymer solution flow through fine pores: influence of pore size on rheological behavior," Journal of Rheology, vol. 26, no. 2, pp. 111-142, 1982.

[5] R. Pit, H. Hervet, and L. Leger, "A sequential quadratic programming algorithm for nonconvex, nonsmooth constrained optimization," Physics Review Letters, vol. 2, no. 85, p. 980, 2000.

[6] E. V. Dose and G. J. Guiochon, "Time scales of transient processes in capillary electrophoresis," Journal of Chromatography, vol. 652, no. 1, pp. 263-275, 1993.

[7] N. G. Green, A. Ramos, A. Gonzalez, H. Morgan, and A. Castellanos, "Fluid flow induced by nonuniform ac electric fields in electrolytes on microelectrodes. III. Observation of streamlines and numerical simulation," Physical Review E, vol. 66, no. 2, Article ID 026305, 2002.

[8] O. Soderman and B. Jonsson, "Electro-osmosis: velocity profiles in different geometries with both temporal and spatial resolution," The Journal of Chemical Physics, vol. 105, no. 23, pp. 1030010311, 1996.

[9] Y. J. Kang, C. Yang, and X. Y. Huang, "Dynamic aspects of electroosmotic flow in a cylindrical microcapillary," International Journal of Engineering Science, vol. 40, no. 20, pp. 2203-2221, 2002.

[10] Y. J. Jian, L. G. Yang, and Q. S. Liu, "Time periodic electroosmotic flow through a microannulus," Physics of Fluids, vol. 22, no. 4, Article ID 042001, 2010.

[11] B. Wiwatanapataphee, Y. H. Wu, J. Archapitak, P. F. Siew, and B. Unyong, "A numerical study of the turbulent flow of molten steel in a domain with a phase-change boundary," Journal of Computational and Applied Mathematics, vol. 166, no. 1, pp. 307319, 2004.

[12] B. Wiwatanapataphee, D. Poltem, Y. H. Wu, and Y. Lenbury, "Simulation of pulsatile flow of blood in stenosed coronary artery bypass with graft," Mathematical Biosciences and Engineering, vol. 3, no. 2, pp. 371-383, 2006.

[13] J. M. Hill, Y. H. Wu, and B. Wiwatanapataphee, "Analysis of flux flow and the formation of oscillation marks in the continuous caster," Journal of Engineering Mathematics, vol. 36, no. 4, pp. 311-326, 1999.

[14] Y. H. Wu, B. Wiwatanapataphee, and M. Hu, "Pressure-driven transient flows of Newtonian fluids through microtubes with slip boundary," Physica A: Statistical Mechanics and Its Applications, vol. 387, no. 24, pp. 5979-5990, 2008.

[15] B. Wiwatanapataphee, Y. H. Wu, M. Hu, and K. Chayantrakom, "A study of transient flows of Newtonian fluids through microannuals with a slip boundary," Journal of Physics A: Mathematical and Theoretical, vol. 42, no. 6, Article ID 065206, 2009.

[16] P. Dutta and A. Beskok, "Analytical solution of time periodic electroosmotic flows: analogies to stokes' second problem," Analytical Chemistry, vol. 73, no. 21, pp. 5097-5102, 2001.

[17] R. J. Hunter, Zeta Potential in Colloid Science: Principles and Applications, Academic Press, New York, NY, USA, 1981. 


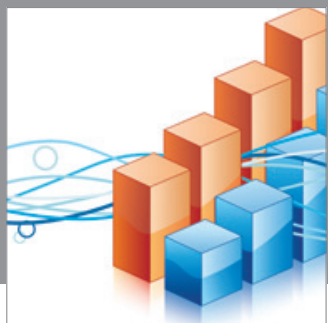

Advances in

Operations Research

mansans

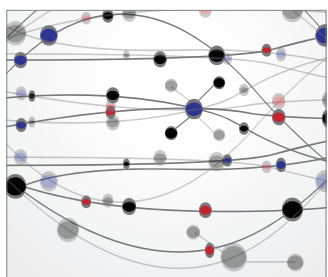

The Scientific World Journal
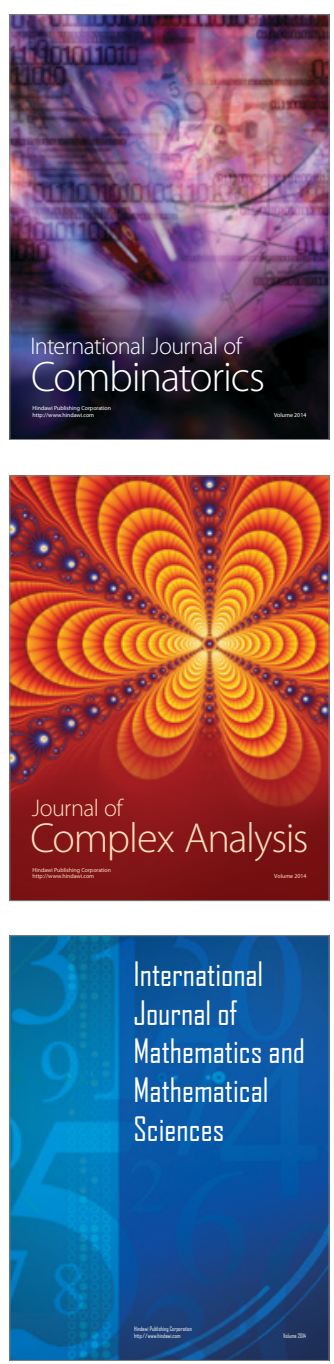
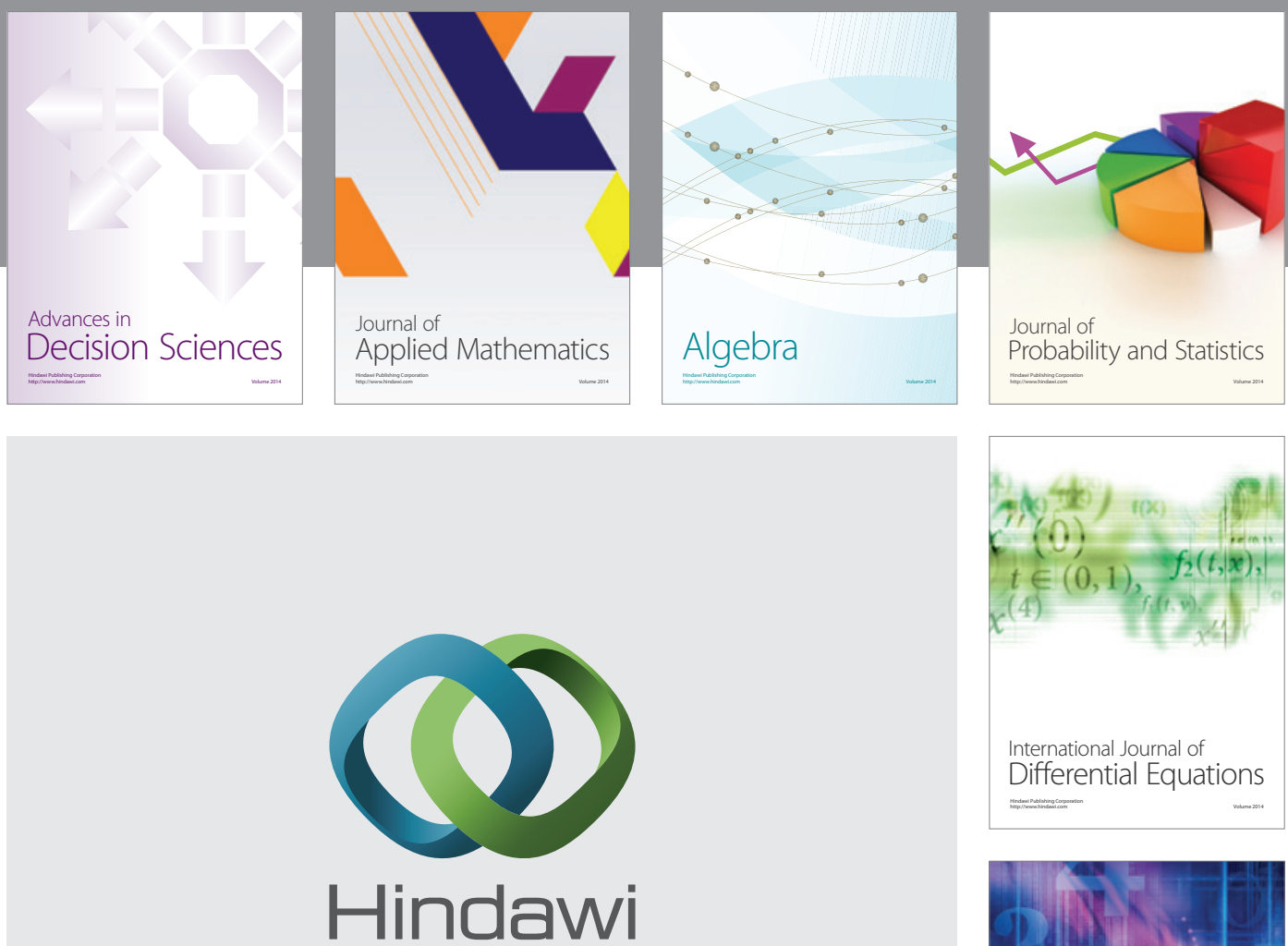

Submit your manuscripts at http://www.hindawi.com
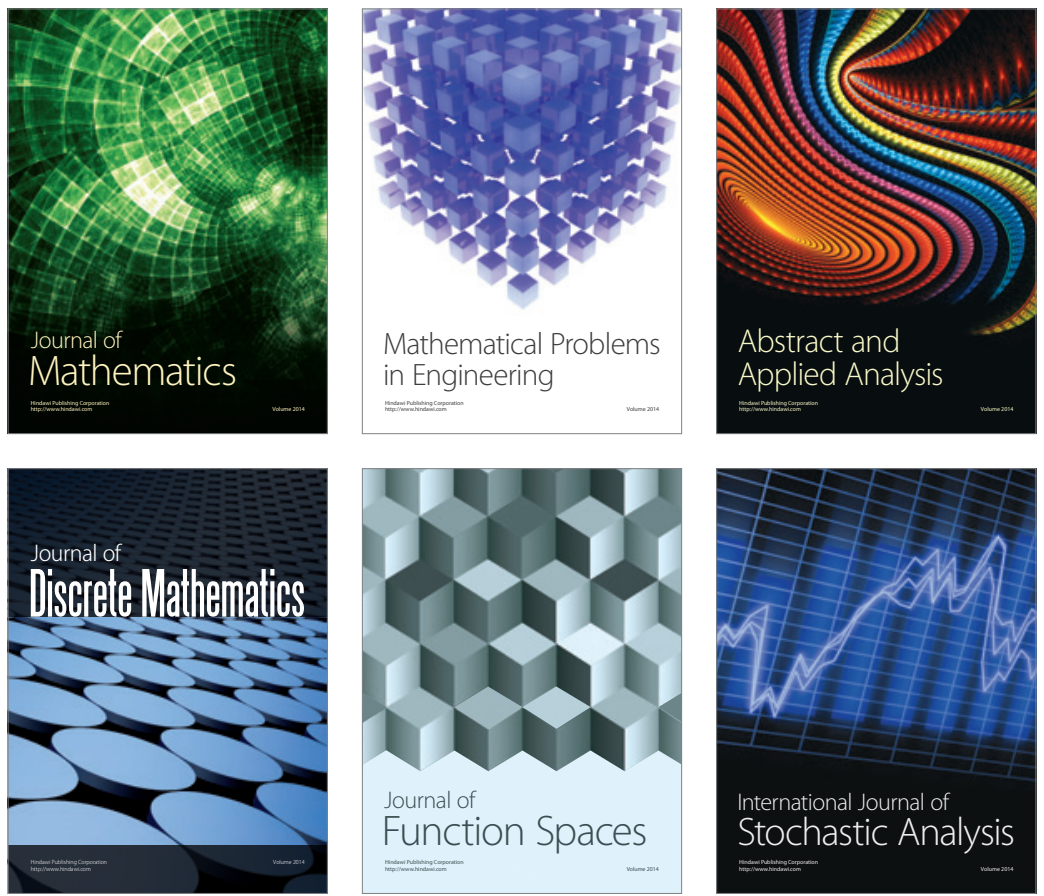

Journal of

Function Spaces

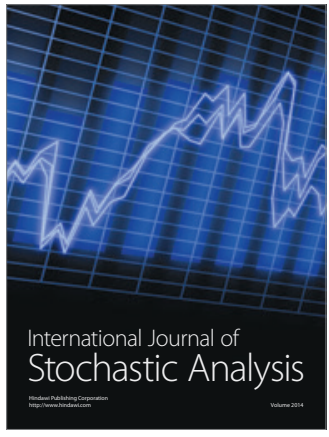

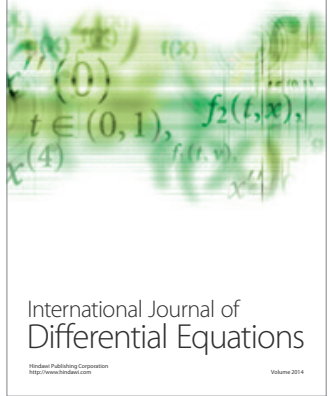
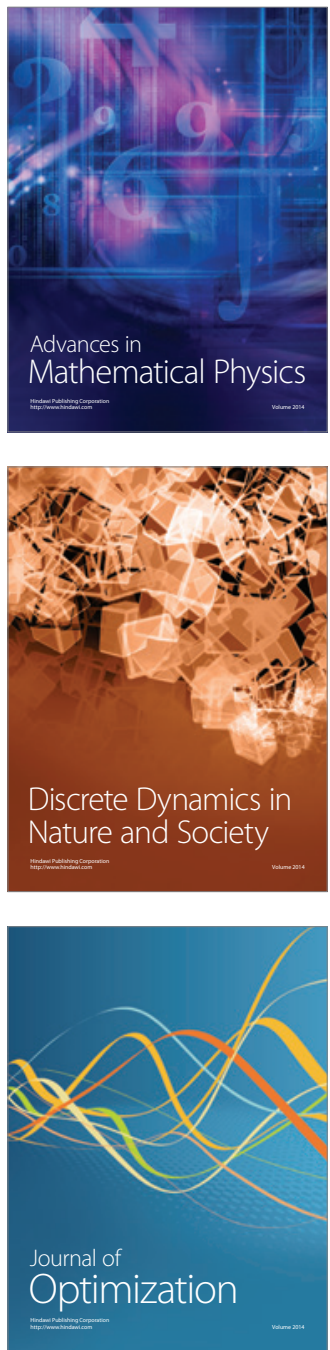